\title{
Efficient Delivery of Therapeutic siRNA with Nanoparticles Induces Apoptosis in Prostate Cancer Cells
}

\author{
Xupeng Mu, ${ }^{1}$ Haibin Lu, ${ }^{2}$ Lianlian Fan, ${ }^{1}$ Shaohua Yan, ${ }^{2}$ and Kebang Hu ${ }^{3}{ }^{3}$ \\ ${ }^{1}$ Department of Central Laboratory, China-Japan Union Hospital, Jilin University, Changchun, Jilin 130021, China \\ ${ }^{2}$ College of Pharmacy, Jilin University, Changchun, Jilin 130021, China \\ ${ }^{3}$ Department of Urology, The First Hospital of Jilin University, Changchun, Jilin 130021, China
}

Correspondence should be addressed to Kebang Hu; kebang_hu@126.com

Received 18 September 2017; Revised 28 January 2018; Accepted 17 February 2018; Published 15 April 2018

Academic Editor: Jorge Pérez-Juste

Copyright (C) 2018 Xupeng $\mathrm{Mu}$ et al. This is an open access article distributed under the Creative Commons Attribution License, which permits unrestricted use, distribution, and reproduction in any medium, provided the original work is properly cited.

\begin{abstract}
Gene silencing using small interfering RNA (siRNA) has shown significant potential in the treatment of cancer. Herein, we developed the lipid-polymer hybrid nanoparticles (PEG-LP/siRNA NPs) for siRNA delivery. The cell viability assay indicated that PEG-LP/siRNA NPs had negligible cell cytotoxicity. The cellular uptake efficiency of PEG-LP/siRNA NPs measured by flow cytometry was up to $94.4 \%$. Importantly, in vitro gene knockdown experiments demonstrated that PEG-LP/siJnk-1 NPs could significantly downregulate the expression of Jnk-1 at both the mRNA and protein levels in DU145 cells. Gene knockdown of Jnk-1 could activate apoptosis in part by the mitochondrial pathway in DU145 cells. Moreover, the PEG-LP/siJnk-1 NPs could inhibit tumor growth in a DU145 xenograft murine model, suggesting its therapeutic promise in cancer therapy.
\end{abstract}

\section{Introduction}

With the capability to silence targeted gene of interest, small interfering RNA (siRNA) has garnered much interest as a potential drug for treatment of various diseases including cancer $[1,2]$. Mutated tumor suppressor genes, oncogenes, and many other genes involved in tumor progression are good targets for gene silencing due to the high specificity of gene silencing by siRNA and lack side effects compared to chemotherapies. Meanwhile, siRNA could target multiple genes of various cellular pathways involved in the growth and proliferation of cancer cells. For cancer treatment, simultaneous silencing of multiple target genes is an effective approach, which could reduce the multiple drugs' resistance caused by overdose of chemotherapy drugs. Meanwhile, gene silencing by siRNA could develop the suitable personalized drugs for the specific patient in the future. However, some deficiencies of siRNA hampered its development in clinical application: fast RNase degradation, poor membrane permeability, and rapid elimination from systemic circulation [3-5]. Therefore, the most pressing mission for transferring siRNA into clinical application is the exploitation of efficient and safe delivery vehicles.

To overcome these obstacles, many approaches including virus-based and nonviral carriers have been used to conjugate or encapsulate siRNA for gene silencing. Virusbased carriers enabled stable gene silencing efficacy in vivo $[6,7]$. However, virus-based carriers were associated with concerns of potential inherent toxicity and immunogenicity $[8,9]$. Recent studies showed that nonviral carriers including liposomes, cationic polymers, or dendrimers could be able to deliver siRNA molecules for gene silencing [10-15]. Among them, polyethylenimine (PEI), the most widely used cationic polymer, has been proven to be an effective nonviral vector for the delivery of siRNA in vitro and in vivo, which was able to load siRNA by electrostatic interaction $[16,17]$. However, the inherent cytotoxicity of PEI hindered its applications when utilizing high molecular weight PEI to deliver siRNA into mammalian cells [18]. To overcome this drawback, several approaches were made such as conjugating with biocompatible or biodegradable polymers or incorporating PEI into the polymer matrix $[19,20]$. As an FDA-approved 
nontoxic and biocompatible polymer, poly(D,L-lactide-coglycolide) (PLGA) has been widely used to encapsulate drugs or genes efficiently $[21,22]$.

The c-Jun $\mathrm{NH}_{2}$-terminal kinases (Jnks) belong to the mitogen-activated protein (MAP) kinase family. Jnk was believed to participate in the important cellular processes such as autophagy, metabolism, DNA-damage response, and apoptosis $[23,24]$. Several studies have reported that the aberrant activation of Jnk could reveal oncogenic features, and sijnk-1 could inhibit the growth and induce apoptosis in some tumor cells such as MCF-7 breast carcinoma cell by mitochondrial caspase-dependent pathway $[25,26]$. Jnk is drawing increasing attention as a promising target of anticancer therapy.

Based on this background, in this study, we aimed to design and synthesize an effective nanocarrier for siRNA delivery. We firstly developed a PLGA polymer core containing PEI/siRNA NPs. And then lipophilic tails of DSPEPEG were self-assembled on the NPs via hydrophobic interactions in order to improve the biocompatibility and prolong the circulation time. The physicochemical properties of the NPs, including siRNA binding capacity and hydrodynamic diameter, were characterized. In vitro toxicity, intracellular uptake, and the antitumor effects of siJnk-1 silencing in a DU145 xenograft murine model were also investigated.

\section{Materials and Methods}

2.1. Materials. Polyethylenimine, ethylenediamine branched $(\mathrm{Mw}=800 \mathrm{Da})(\mathrm{PEI})$, ester-terminated poly(D,L-lactideco-glycolide) (PLGA, viscosity of $0.26-0.54 \mathrm{dL} / \mathrm{g}$ ), and 4,6diamidino-2-phenylindole (DAPI) were purchased from Sigma-Aldrich (MO, USA). 1,2-Distearoyl-sn-glycero-3-phosphoethanolamine-N-[methoxy(polyethyleneglycol)-3000] (DSPE-PEG) with PEG molecular weight 3000 was purchased from Seebio Biological (Shanghai, China). SiRNA targeting Jnk-1 (sense: $5^{\prime}$-GCGACGACCUUACAGAGCGUU-3'; antisense: $5^{\prime}$-CGCUCUGUAAGGUCG UCGCUU-3'; ${ }^{\prime}$ named siJnk-1), negative control siRNA (sense: $5^{\prime}$-UUCUCCGAA CGUGUCACGUdTdT-3'; named siN.C.), and fluorescein- (FAM-) labeled siRNA (sense: $5^{\prime}$ UUCUCCGAACGUGUCACGUTT-3'; named FAMsiRNA) were acquired from GenePharma Co., Ltd. (Shanghai, China). Anti-pro-caspase-3, anti-pro-caspase-9, anti-Jnk-1, anti-bax, anti-bcl-2, anti-cytochrome $\mathrm{C}$, and anti- $\beta$-actin were purchased from Santa Cruz Biotechnology Inc. (CA, USA). IRDye ${ }^{\circledR} 680$ goat anti-mouse IgG and IRDye 680 goat anti-rabbit IgG were purchased from Li-COR Biosciences Inc. (NE, USA). All other chemical reagents including tetrahydrofuran (THF) were purchased from Guangzhou Chemical Reagent Factory (Guangdong, China).

2.2. Cell and Animals. Human prostate cancer DU145 cell lines were obtained from ATCC (VA, USA). All cell lines were maintained in DMEM supplemented with 10\% fetal bovine serum (FBS), $100 \mathrm{mg} \mathrm{mL}^{-1}$ streptomycin, and $100 \mathrm{U} \mathrm{mL}^{-1}$ penicillin and incubated in a humidified atmosphere of $5 \%$ $\mathrm{CO}_{2}$ at $37^{\circ} \mathrm{C}$.

$\mathrm{BALB} / \mathrm{c}$ nude mice (4-5 weeks old) were purchased from Vital River Company (Beijing, China) and were maintained under specific pathogen-free conditions in plastic cages. The animals were treated according to the ethical guidelines of Jilin University after obtaining approval from the Animal Care and Use Committee of Jilin University. All animal experiments were performed in compliance with the internationally accepted animal care guidelines.

2.3. Preparation of Lipid-Polymer Hybrid (PEG-LP/siRNA) NPs. The siRNA-encapsulated lipid-polymer hybrid NPs were synthesized by a simple self-assembly method. Firstly, $4 \mathrm{mg}$ PLGA and $0.8 \mathrm{mg}$ cationic polymer PEI were dissolved in $1 \mathrm{~mL}$ THF solvent. Then $50 \mathrm{uL}$ siRNA $(4 \mathrm{nmol})$ was added to THF solution to form PEI/siRNA nanocomplexes. Next, the reaction mixture was added dropwise into a $15 \mathrm{~mL}$ aqueous solution containing DSPE-PEG under stirring until the NPs formed. The residual THF in the suspension was evaporated by continuously stirring at room temperature. Then, the prepared NPs were collected using $100 \mathrm{kDa}$ MWCO Amicon ultratubes (Millipore, MA, USA) and concentrated in PBS solution.

2.4. Characterization of PEG-LP/siRNA NPs. The morphology of prepared NPs was characterized using transmission electron microscope (TEM) (Hitachi H-800 Hitachi, Tokyo, Japan) operated at $80 \mathrm{kV}$. The size and its distribution of prepared NPs were assessed by Dynamic Light Scattering (DLS) (Malvern Zetasizer Nano Instrument, Malvern, $\mathrm{UK})$.

2.5. Gel Retardation Assay. Gel retardation assay was used to assess the siRNA loading ability of the PEG-LP/siRNA NPs. PEG-LP/siRNA NPs were prepared at different N/P ratios (molar ratios of cationic amino groups of PEI to phosphate groups of siRNA) ranging from 0 to 10 (siRNA concentration was fixed at $100 \mathrm{nM}$ ). After forming nanocomplexes as described above, $20 \mathrm{uL}$ formed PEG-LP/siRNA NPs was loaded into $1 \%$ agarose gel containing $0.5 \mathrm{ug} \mathrm{mL}$ ethidium bromide. Electrophoresis was performed at $100 \mathrm{~V}$ in $1 \mathrm{x}$ TAE buffer ( $\mathrm{pH} 8.3$, containing $40 \mathrm{mM}$ Tris base, $20 \mathrm{mM}$ acetic acid, $1 \mathrm{mM}$ EDTA) for $20 \mathrm{~min}$ and visualized under a UV illuminator (Bio-Rad Laboratories, CA, USA).

2.6. siRNA Encapsulation Efficiency. The encapsulation efficiency of siRNA in the PEG-LP/siRNA NPs was evaluated by measuring the concentration of unencapsulated siRNA in the supernatant. In brief, nanocomplexes prepared with FAM-siRNA were centrifuged at $15,000 \mathrm{rpm}$ for $10 \mathrm{~min}$, and then the fluorescence intensity of FAM-siRNA $(E x / E m=480 / 520 \mathrm{~nm})$ in supernatant was measured using a BioTek Synergy HT Multi-Mode Microplate Reader (BioRad Laboratories, CA, USA). The encapsulation efficiency of siRNA was calculated as follows: encapsulation efficiency 
$(\%)=\left(F_{0}-F_{1}\right) / F_{0} \times 100 \%$, where $F_{0}$ and $F_{1}$ were the fluorescence intensity of siRNA used for encapsulation and in the supernatant, respectively.

2.7. Cellular Uptake Efficiency of PEG-LP/siRNA NPs. Cellular uptake efficiency of siRNA in vitro was quantitatively evaluated by flow cytometry. DU145 cells $\left(2 \times 10^{5}\right.$ cells/well $)$ were seeded in 6-well plates and incubated overnight. Then, the medium was replaced with fresh DMEM medium which contained various FAM-siRNA formulations including naked siRNA, PEG-LP/siRNA NPs, or lipofectamine 2000/siRNA (Lipo2K/siRNA) at siRNA concentration of $10 \mathrm{nM}$. After $6 \mathrm{~h}$ incubation, cells were washed with PBS, trypsinized, harvested by centrifugation, and resuspended in PBS. For each run, the fluorescence of FAM-siRNA per $1 \times 10^{5}$ cells was analyzed on a FACS Calibur flow cytometer (BD Biosciences, NJ, USA). The proportion of FAM positive cells in each run was equivalent to the cellular uptake efficiency of siRNA. The untreated cells were used as the control.

2.8. Intracellular Distribution of PEG-LP/siRNA NPs. Confocal laser scanning microscopy (CLSM) was employed to study the intracellular distribution of PEG-LP/siRNA NPs. In brief, DU145 cells $\left(1 \times 10^{5}\right.$ cells/well $)$ were seeded into confocal dishes and incubated overnight. Then, PEG-LP/siRNA NPs containing FAM-siRNA $(10 \mathrm{nM})$ was added. After incubation for $6 \mathrm{~h}$ or $24 \mathrm{~h}$, cells were washed with PBS, fixed in $4 \%$ paraformaldehyde. To label the cell nucleus, samples were incubated with $1 \mathrm{~g} \mathrm{~mL}^{-1}$ DAPI (Beyotime, Jiangsu, China) for 10 min in PBS, followed by rinsing with PBS. Intracellular distribution of FAM-siRNA was observed with an Olympus FluoView 500 confocal microscope (Olympus Corporation, Tokyo, Japan).

2.9. In Vitro Cytotoxicity of PEG-LP/siN.C. NPs. The CCK-8 assay was used to assess the cytotoxicity of PEG-LP/siN.C. NPs. Firstly, DU145 cells were seeded at $1 \times 10^{4}$ cells per well in a 96-well plate and incubated overnight at $37^{\circ} \mathrm{C}$. The medium of each well was replaced with fresh DMEM medium containing PEG-LP/siN.C. NPs or PEG-LP/siJnk-1 NPs at a series of siRNA concentrations $(1 \mathrm{nM}, 5 \mathrm{nM}, 10 \mathrm{nM}$, $25 \mathrm{nM}$, and $50 \mathrm{nM}$ ). After $24 \mathrm{~h}$ incubation, $200 \mathrm{uL}$ medium was removed and $10 \mu \mathrm{L}$ of CCK-8 was added to each well for another $2 \mathrm{~h}$ treatment. Finally, the absorbance was read on an ELx-800 Microplate Reader (BioTek Instruments, VT, USA) at $450 \mathrm{~nm}$. The cell viability (\%) was calculated as follows: cell viability $(\%)=\mathrm{OD}_{450 \text { (sample) }} / \mathrm{OD}_{450 \text { (control) }} \times 100 \%$, where $\mathrm{OD}_{450 \text { (sample) }}$ and $\mathrm{OD}_{450 \text { (control) }}$ were the absorbance of the test sample and the control, respectively. Experiments were performed in triplicate and the decrease of cell viability represented the increase of cell cytotoxicity.

2.10. Cell Apoptosis Analysis In Vitro. DU145 cells cultured in 6-well plates were treated with PEG-LP/sijnk-1 NPs or PEG-LP/siN.C. NPs (siRNA concentration of $50 \mathrm{nM}$ ). After treatment for $72 \mathrm{~h}$, the cell suspension was mixed thoroughly with $5 \mathrm{uL}$ of fluorescein isothiocyanate-labeled Annexin $\mathrm{V}$ (Annexin V-FITC) and $5 \mathrm{uL}$ of propidium iodide (PI) (Proteintech Group, Inc., Hubei, China) for $15 \mathrm{~min}$ in the dark. Apoptotic cells were detected by flow cytometer (BD Biosciences, NJ, USA).

2.11. In Vitro Gene Silencing with PEG-LP/siJnk-1 NPs. Quantitative real-time PCR (qRT-PCR) was used to assess Jnk1 silencing effects after PEG-LP/siJnk-1 NPs treatment. DU145 cells were transfected with PBS, naked siJnk-1, PEG-LP/siN.C. NPs, Lipo2K/siJnk-1, or PEG-LP/siJnk-1 NPs (siJnk-1 concentration of $10 \mathrm{nM}$ ). After $24 \mathrm{~h}$ incubation, total RNA from cells was extracted using RNeasy mini kit (Qiagen, Duesseldorf, Germany) according to the manufacturer's instructions. 2 ug RNA was reverse-transcribed into cDNA using the PrimeScript ${ }^{\mathrm{TM}}$ 1st Strand cDNA Synthesis Kit (Takara, Otsu, Japan). Then, qRT-PCR was performed using SYBR Green PCR reagents on an ABI PCR System 9700 (Applied Biosystems, CA, USA). The amplification procedure was as follows: $95^{\circ} \mathrm{C}, 10 \mathrm{~min} ; 94^{\circ} \mathrm{C}, 30 \mathrm{sec} ; 60^{\circ} \mathrm{C}$, $30 \mathrm{sec} ; 72^{\circ} \mathrm{C}, 45 \mathrm{sec} ; 40$ cycles. The primers used in the PCR reactions were as follows: Jnk-1-forward, CGTCTGGTGGAAGGAGAGAG, Jnk-1-reverse, TAATAACGGGGGTGGAGGAT; and $\beta$-actin-forward, GAAATCGTGCGTGACATCAAAG, $\beta$-actin-reverse, TGTAGT TTCATGGATGCCACAG. All of the gene expression values were normalized to those of $\beta$-actin and calculated using the ${ }^{\Delta \Delta} \mathrm{Ct}$ method. All reactions were performed in triplicate.

2.12. Western Blot Analysis. DU145 cells were treated as described for the qRT-PCR analysis and were harvested at $72 \mathrm{~h}$ after transfection. Then the cells were washed with cold PBS and resuspended in $100 \mathrm{uL}$ of lysis buffer ( $\mathrm{pH} 7.4$, containing $50 \mathrm{mM}$ Tris-HCl, $1 \%$ SDS, $1 \mathrm{mM}$ EDTA, $1 \mathrm{mM}$ PMSF, $1 \%$ Triton X-100). The cell lysates were incubated on ice for $30 \mathrm{~min}$, and the supernatants were collected after centrifugation at $12,000 \mathrm{rpm}$ for $10 \mathrm{~min}$ at $4^{\circ} \mathrm{C}$. Protein concentration was determined using a BCA Protein Assay Kit according to the protocol (Beyotime Inc., Jiangsu, China). Total protein $(100 \mathrm{ug})$ was separated on 10\% SDS-PAGE and then transferred onto nitrocellulose membranes (Millipore, MA, USA) at $200 \mathrm{~mA}$. After blocking with $5 \%$ nonfat dry milk in TBST (pH 7.4, containing $50 \mathrm{mM}$ Tris- $\mathrm{HCl}, 150 \mathrm{mM}$ $\mathrm{NaCl}, 0.1 \%$ Tween-20) for $1 \mathrm{~h}$, the membranes were incubated with anti-pro-caspase-3, anti-pro-caspase-9, anti-Jnk1 , anti-bax, anti-bcl-2, anti-cytochrome $\mathrm{C}$, or anti- $\beta$-actin monoclonal antibody $(1: 1000)$ overnight at $4^{\circ} \mathrm{C}$, respectively. The membranes were further incubated with IRDye 680 goat anti-mouse IgG or anti-rabbit IgG secondary antibody $(1: 10,000)$ for $3 \mathrm{~h}$ at room temperature and visualized using an Odyssey infrared imaging system (LI-COR Biosciences, NE, USA).

2.13. In Vivo Tumor Cell Apoptosis and Therapeutic Efficacy. To establish the prostate cancer xenograft tumor model, $1 \times 10^{7}$ DU145 cells in $100 \mathrm{uL}$ PBS were subcutaneously injected into the backs of BALB/c nude mice. When the tumor volume $\left(V ; \mathrm{mm}^{3}\right)$ reached approximately $50 \mathrm{~mm}^{3}$ 

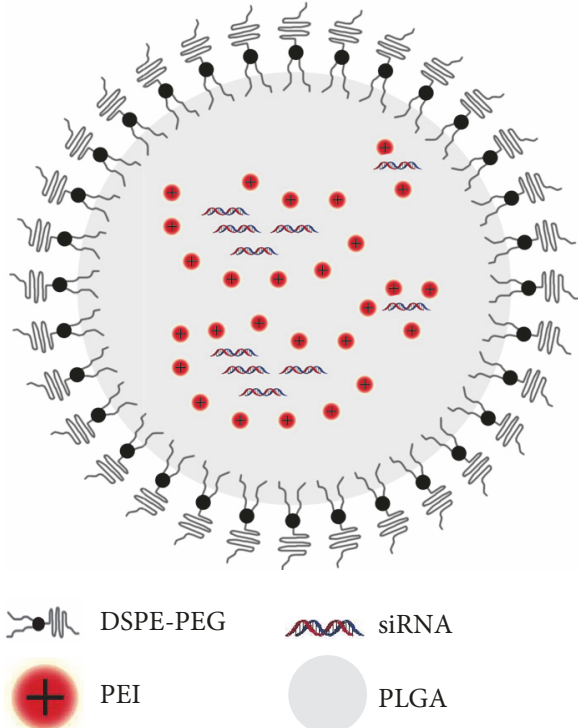

(a)

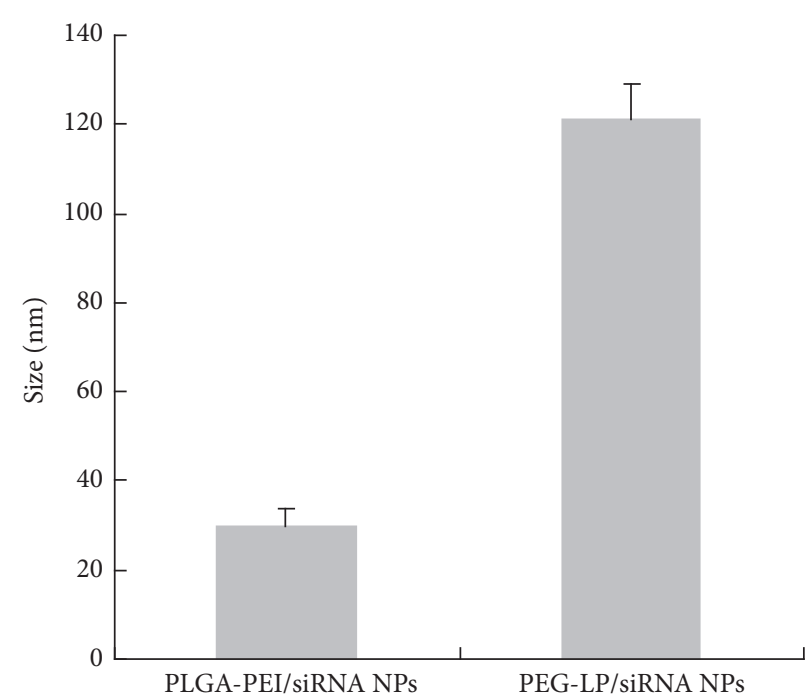

(b)

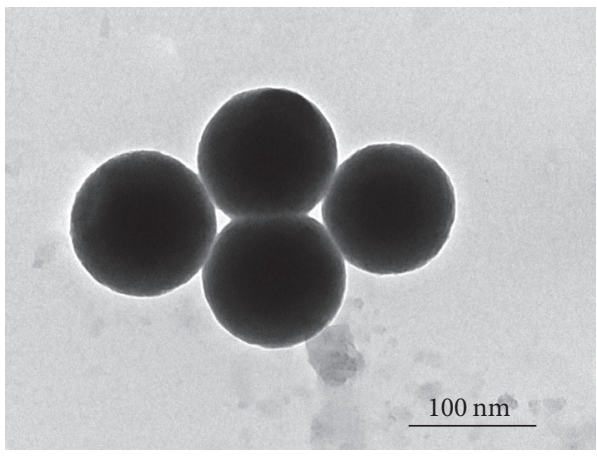

(d)

FIGURE 1: Characterization of the PEG-LP/siRNA NPs. (a) Schematic diagram of the prepared NPs structure. (b) Mean particle size of the prepared NPs measured by DLS. (c) Gel retardation assay of siRNA complexed with nanoparticles at different N/P ratios. (d) Transmission electron microscopic image of prepared NPs.

$\left(V=1 / 2 \times\right.$ width $^{2} \times$ length, measured using a caliper $)$, mice were randomly divided into 4 groups (five mice per group). Animals were treated with PBS, PEG-LP NPs, PEG-LP/siN.C. NPs, or PEG-LP/siJnk-1 NPs by intravenous injection at a dose of 600 ug siRNA per kg of mouse weight every other day for 20 days. Tumor sizes were measured every 4 days. Four days after the last administration, the mice were sacrificed and tissues including heart, liver, spleen, lung, kidney, tumors were collected for further analysis.

2.14. Immunohistochemical Analysis. Tumor tissues and other major organs (heart, liver, spleen, lung, and kidney) were fixed in $4 \%$ paraformaldehyde, embedded in paraffin, and sectioned for analysis after various treatments. The frozen tissue slides were further stained with hematoxylin and eosin (HE) following the standard protocol and examined using an Olympus IX5-RFACA fluorescence microscope (Olympus Corporation, Tokyo, Japan).
Apoptosis in the tumor sections following various treatments was analyzed using the terminal deoxyribonucleotidyl transferase- (TdT-) mediated dUTP nick-end labeling (TUNEL) assay according to the manufacturer's suggested protocol (Roche, Basel, Switzerland).

2.15. Statistical Analysis. All data were presented as the mean \pm standard deviation (SD) and analyzed by using Student's $t$ test. A value of $p<0.05$ was considered statistically significant.

\section{Results and Discussion}

3.1. Preparation and Characterization of PEG-LP/siRNA NPs. The siRNA-encapsulated lipid-polymer hybrid NPs (PEGLP/siRNA NPs) were prepared using a simple self-assembly precipitation method. Figure 1(a) illustrated the structure of the lipid-polymer hybrid NPs. Firstly, aqueous siRNA was 
mixed with THF solution containing cationic polymer PEI and PLGA polymer in a 1:50 volume ratio. The cationic polymer PEI could bind the negatively charged siRNA and then self-assembled quickly into PEI/siRNA nanocomplexes (PEI/siRNA) with a size of about $30 \mathrm{~nm}$ (Figure 1(b)). Subsequently, the prepared composite materials in THF solution were rapidly added to an aqueous solution containing DSPEPEG. As expected, the PLGA polymer and PEI/siRNA complex were coprecipitated to form the polymeric NPs, which should be composed of PLGA-PEI/siRNA inner core and a DSPE-PEG outer shell.

To determine if the lipid-polymer hybrid NPs could complex negatively charged siRNA, a gel retardation assay was conducted after incubating the nanoparticles with siRNA $(100 \mathrm{nM})$ at different N/P ratios. As shown in Figure 1(c), PEG-LP/siRNA NPs were able to encapsulate siRNA in a dose-dependent manner with complete complexation at an $\mathrm{N} / \mathrm{P}$ ratio of 10 or greater.

The morphology of the PEG-LP/siRNA NPs formed at an N/P ratio of 10 was observed by TEM. The nanocomplexes exhibited a compact and spherical morphology with a narrow size distribution (Figure 1(d)). Meanwhile, the DLS results showed that the diameter of PEG-LP/siRNA NPs was about $120 \mathrm{~nm}$ (Figure 1(b)), which was consistent with that of TEM results. The siRNA encapsulation efficiency was about $70 \%$ at $\mathrm{N} / \mathrm{P}$ ratio of 10 (data not shown). The excellent siRNA binding ability of PEG-LP/siRNA NPs might be due to the fact that the cationic amine groups of PEI assisted the hybrid NPs in retaining the siRNA [27].

3.2. In Vitro Cellular Uptake Efficiency and Intracellular Distribution. High siRNA uptake of NPs is a prerequisite for gene silencing. FAM-siRNA was used as a fluorescent probe to monitor the siRNA uptake efficiency of PEG-LP/siRNA in DU145 cells by flow cytometric analysis. As shown in Figure 2(a), for naked siRNA, only few FAM-labeled siRNA penetrated into the cells, and the cellular uptake efficiency was negligible. However, for PEG-LP/siRNA NPs, the cellular uptake efficiency was up to $94.4 \%$ which was slightly higher than the commercial transfection agent lipofectamine 2000 (88.0\%).

Intracellular distribution of FAM-labeled siRNA in DU145 cells was evaluated by CLSM observation. As shown in Figure 2(b), it was clearly demonstrated that FAM-siRNA had been internalized and observed in the cytoplasma of DU145 cells $6 \mathrm{~h}$ after transfection. After incubation for $24 \mathrm{~h}$, most of the green fluorescence FAM-siRNA was diffusely distributed in cytoplasm and even cell nucleus. Meanwhile, the fluorescence signal of FAM-siRNA was getting stronger. The results provided strong evidence showing that PEG-LP/siRNA NPs exhibited excellent cellular internalization.

3.3. In Vitro Cytotoxicity and Apoptosis Evaluation of PEGLP/siRNA NPs. The cytotoxicities of the PEG-LP/siN.C. NPs at a series of siRNA concentrations $(1 \mathrm{nM}, 5 \mathrm{nM}, 10 \mathrm{nM}$, $25 \mathrm{nM}$, and $50 \mathrm{nM}$ ) were tested by CCK- 8 assay. As shown in Figure 3(a), nearly $100 \%$ of DU145 cells remained viable after $24 \mathrm{~h}$ incubation with PEG-LP/siN.C. NPs when the final concentration of siN.C. was $50 \mathrm{nM}$ or less. The results suggested that the PEG-LP/siN.C. NPs possessed a lower cytotoxicity which was perfect for in vivo application. In contrast, the cell viabilities were decreased when DU145 cells were incubated with PEG-LP/siJnk-1 NPs at the concentration of $25 \mathrm{nM}$ or greater. That is to say, the cytotoxicity effects of PEG-LP/siJnk-1 NPs mainly come from siRNA targeting Jnk-1.

In order to find whether the cytotoxicity effects of PEGLP/siJnk-1 NPs derived from the increase in cell apoptosis, the apoptosis of DU145 cells was detected using flow cytometric. As shown in Figure 3(b), PEG-LP/siJnk-1 NPs significantly increased the portion of apoptosis cells versus the PEG-LP/siN.C. NPs (15.4\% versus $1.4 \%$ ) after a $72 \mathrm{~h}$ treatment. The results showed that PEG-LP/siJnk-1 NPs could induce apoptosis of DU145 cells by Jnk-1 gene silencing.

3.4. Analysis of qRT-PCR and Western Blot for Gene Knockdown. The ideal siRNA delivery system should have the ability not only to deliver siRNA but also to significantly reduce the expression of its target genes. To assess the gene knockdown efficiency of PEG-LP/siJnk-1 NPs, the expression of Jnk-1 in DU145 cells treated with PEG-LP/siJnk-1 NPs was analyzed by qRT-PCR and western blot. As shown in Figure 4(a), decreased expression of Jnk-1 mRNA was observed in cells treated with Lipo2K/siJnk-1 and PEGLP/siJnk-1 NPs compared with the control group (PBS). Notably, PEG-LP/siJnk-1 NPs lowered the Jnk-1 mRNA expression approximately up to $55 \%$ from its control group. Meanwhile, the results showed that naked siInk-1, and PEGLP/siN.C. NPs could not reduce the expression of Jnk-1 mRNA. Western blot analysis showed that treatment with PEG-LP/siJnk-1 NPs but not with PEG-LP/siN.C. led to markedly decreased Jnk-1 protein expression in DU145 cells and the knockdown efficiency was consistent with the mRNA expression data (Figure 4(b)).

3.5. The Apoptotic Mechanism of Jnk-1 Knockdown In Vitro. To further probe the mechanisms through which Jnk1 knockdown may cause the apoptosis of DU145 cells, we focused on members of the cysteine aspartate-specific proteases (caspases) protein family which were important mediators of apoptosis signaling and played important roles during the apoptosis phases [28]. Several apoptosis-related proteins including caspases- 3 and caspases- 9 were studied. The western blotting results indicated that PEG-LP/siJnk1 NPs could downregulate the expression of Bcl-2 and upregulate the expression of Bax, which successively led to the release of cytochrome $\mathrm{C}$ from the mitochondria and then the degradation of pro-caspase- 9 and pro-caspase3 and ultimately resulted in cell apoptosis (Figure 5). All the results suggested that siJnk- 1 could induce apoptosis at least partly by the mitochondrial caspase-dependent pathway. 


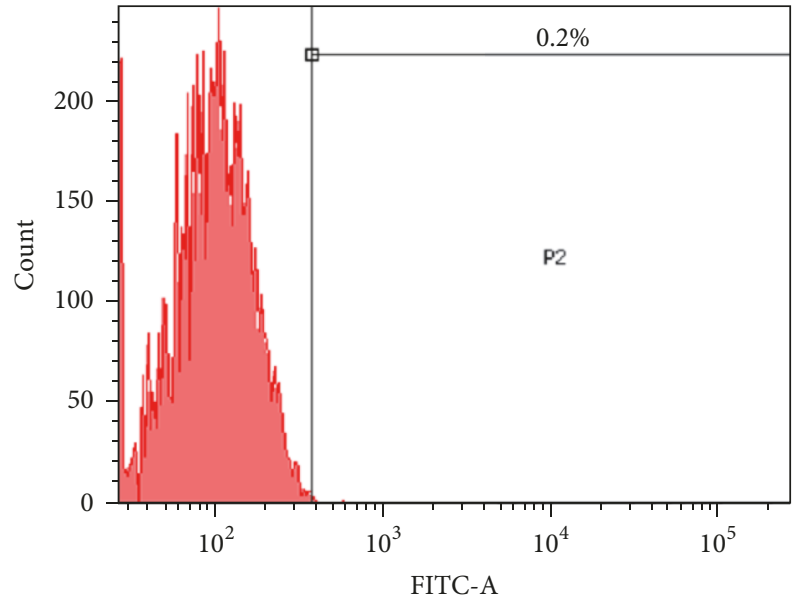

Thed siRNA

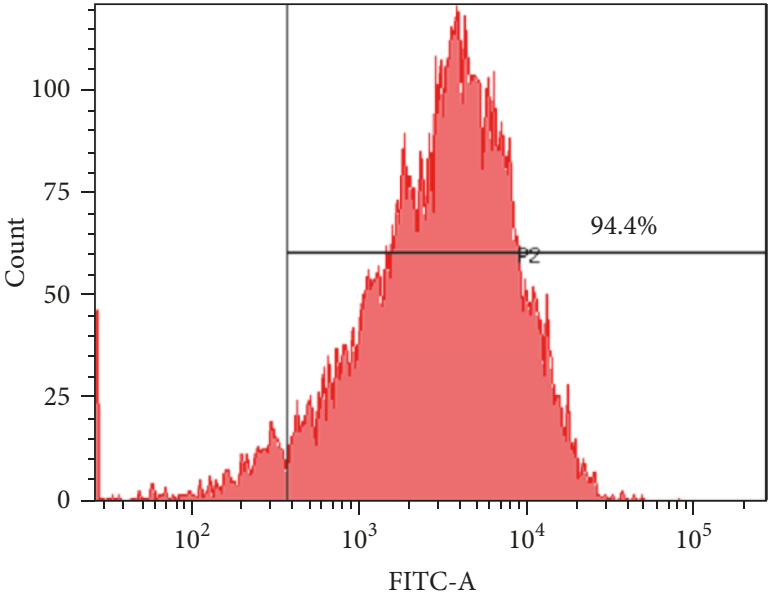

PEG-LP/siRNA NPs

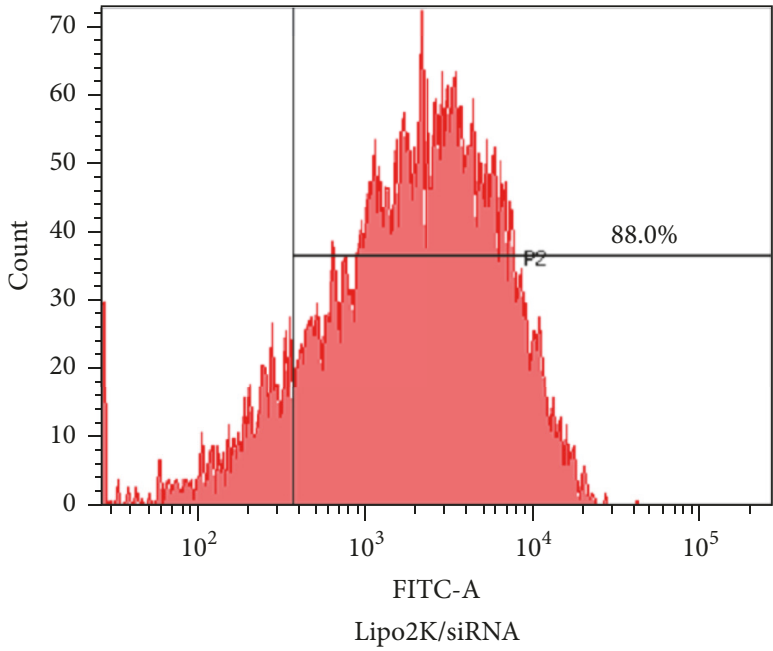

(a)
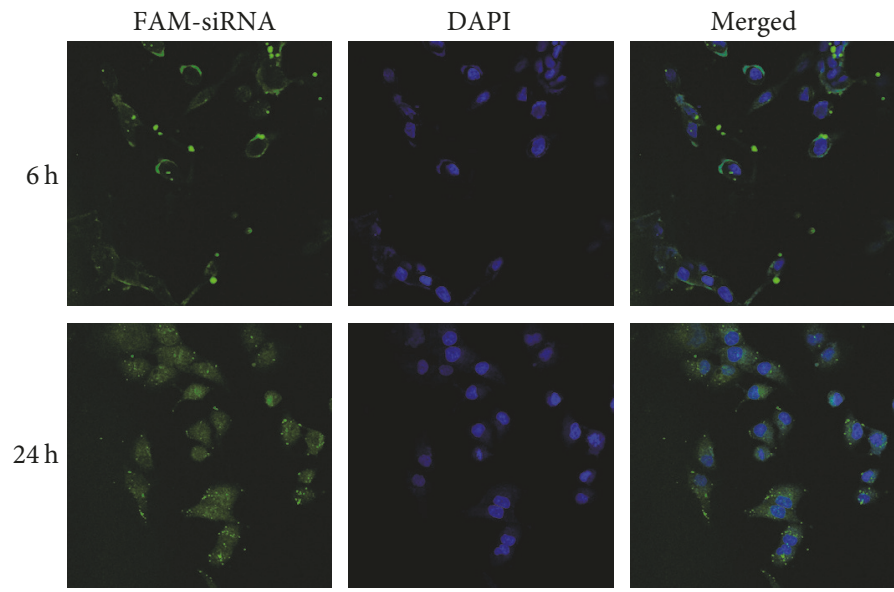

(b)

FIGURE 2: In vitro cell uptake efficiency and intracellular distribution. (a) The uptake efficiency of fluorescence-labeled PEG-LP/siRNA in DU145 cells measured by flow cytometric analysis. (b) Intracellular distribution of fluorescence-labeled PEG-LP/siRNA NPs in DU145 cells at different time points. Green, fluorescence-siRNA; blue, DAPI stained nucleus. 


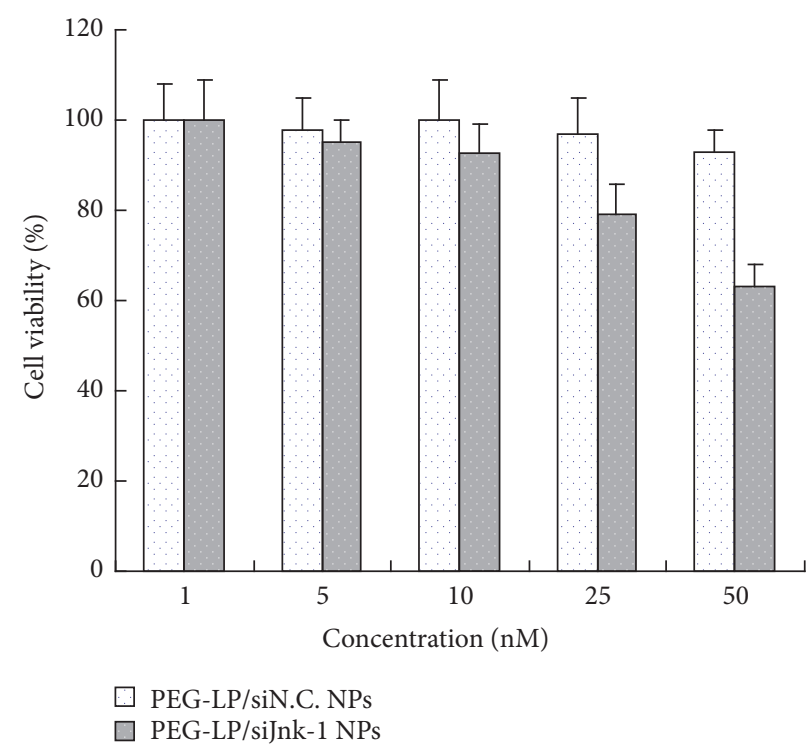

(a)
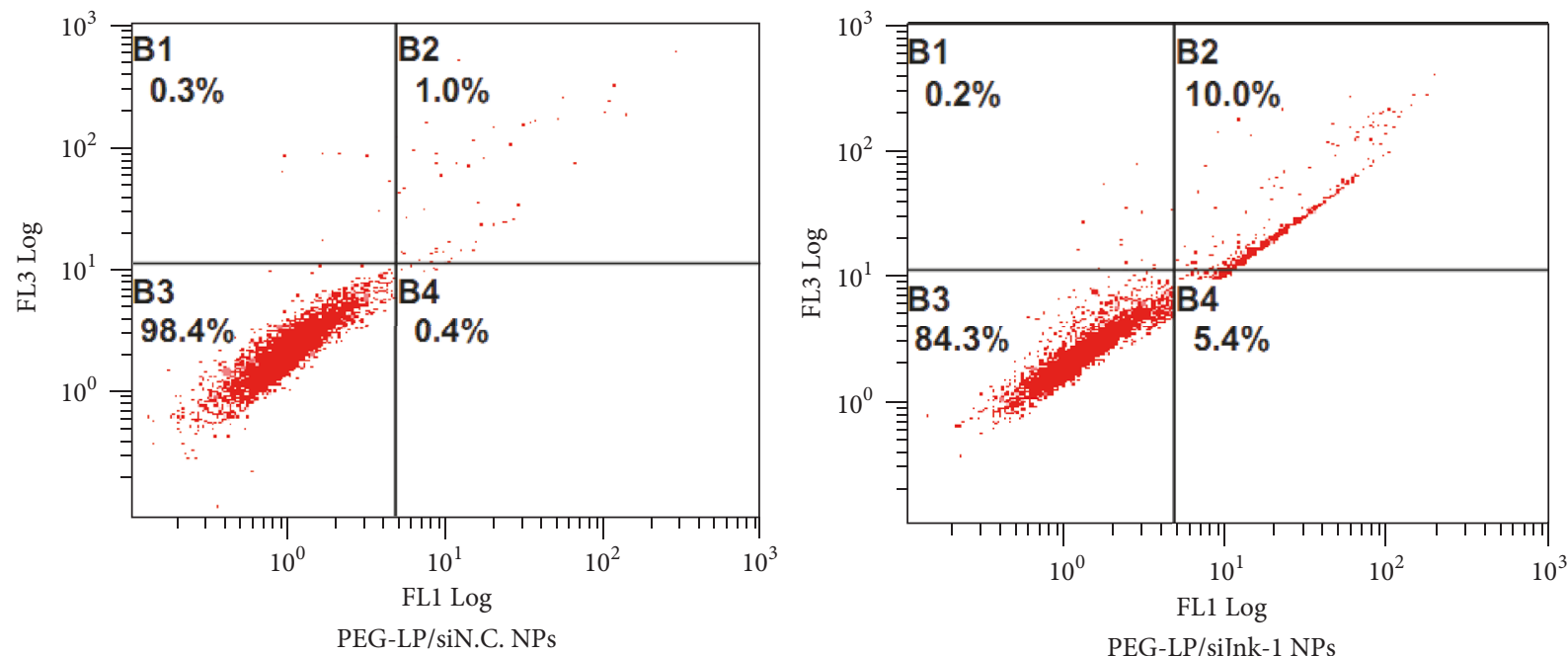

(b)

FIGURE 3: In vitro cytotoxicity and apoptosis evaluation of PEG-LP/siRNA NPs. (a) Cytotoxicity of PEG-LP/siN.C. NPs or PEG-LP/siJnk-1 NPs at different siRNA concentrations after $48 \mathrm{~h}$ incubation. (b) The apoptosis of DU145 cells treated by PEG-LP/siN.C. NPs or PEG-LP/siJnk-1 NPs was detected using flow cytometric.

3.6. In Vivo Tumor Growth Suppression following Systemic Administration. To investigate the tumor therapeutic efficiency following systemic administration of PEG-LP/siJnk1 NPs in vivo, we used a DU145 subcutaneous xenograft model to monitor tumor progression. Each mouse received various formulations at a dose of $600 \mathrm{ug}$ siJnk-1 per $\mathrm{kg}$ mouse weight via the tail vein injection every other day. As shown in Figure 6(a), there was no significant difference on tumor growth among mice treated with PEG-LP/siN.C. NPs compared to PBS. In contrast, the PEG-LP/siJnk-1 NPs group showed significant inhibition on tumor growth. These results were further validated by TUNEL assay analysis in which apoptosis induced by PEG-LP/siJnk-1 NPs treatment was increased compared with PBS group and PEG-LP/siN.C.
NPs group (Figure 6(b)). These results suggested that PEGLP/siJnk-1 NPs could suppress tumor growth by inducing apoptosis in DU145 tumor cells.

Finally, the potential toxicity of PEG-LP/siJnk-1 NPs in vivo was further investigated by $\mathrm{HE}$ staining. The results showed that no obvious histopathological abnormalities and tissue damage were noticed for mice of all experimental groups at the tested dose (Figure 6(c)).

\section{Conclusions}

We have developed a novel lipid-polymer hybrid NPs for the systemic delivery of siRNA, using a biocompatible and 


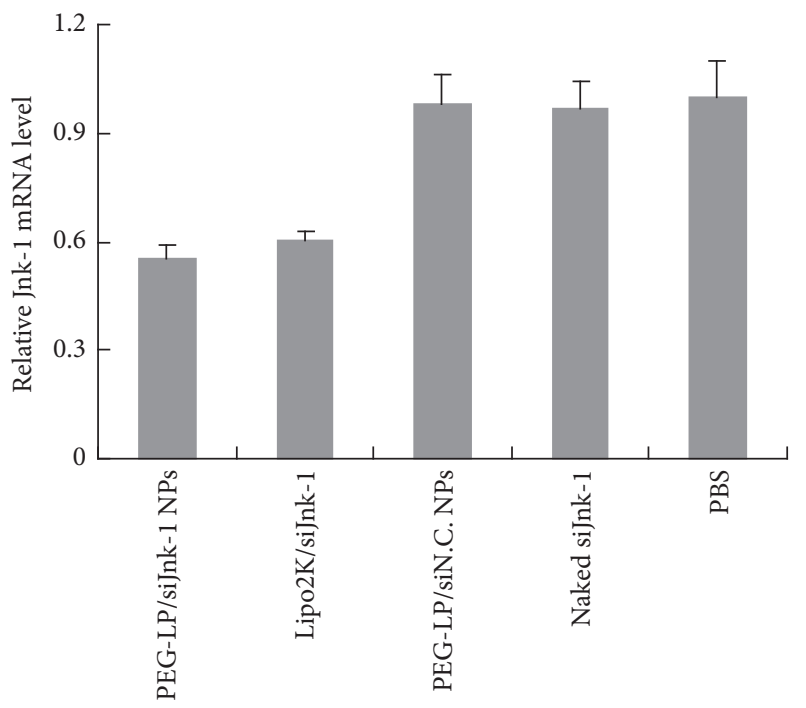

(a)

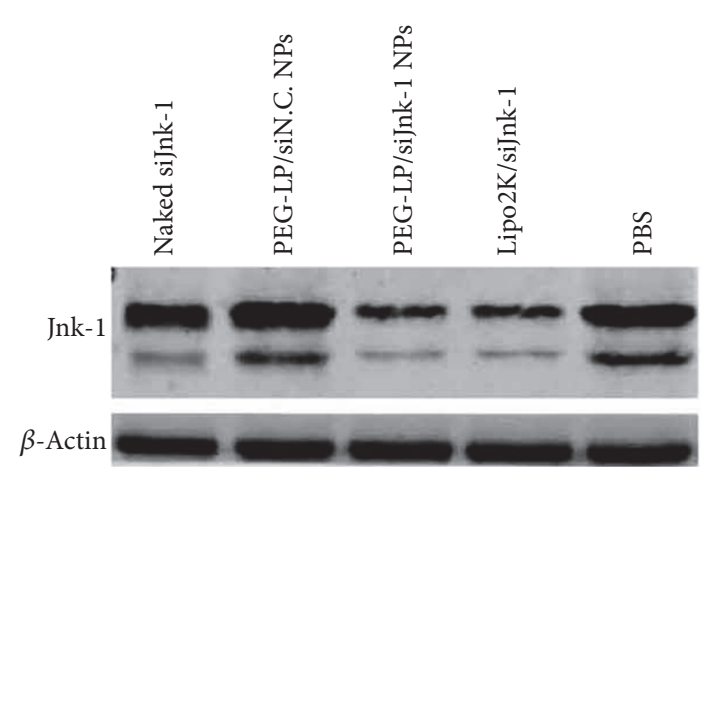

(b)

FIGURE 4: In vitro gene silencing efficiency on DU145 cells. Relative Jnk-1 mRNA (a) and Jnk-1 protein (b) expression of DU145 cells treated with PBS, naked siJnk-1, PEG-LP/siN.C. NPs, Lipo2K/siJnk-1, or PEG-LP/siJnk-1 NPs, which were determined by qRT-PCR and western blot analysis.

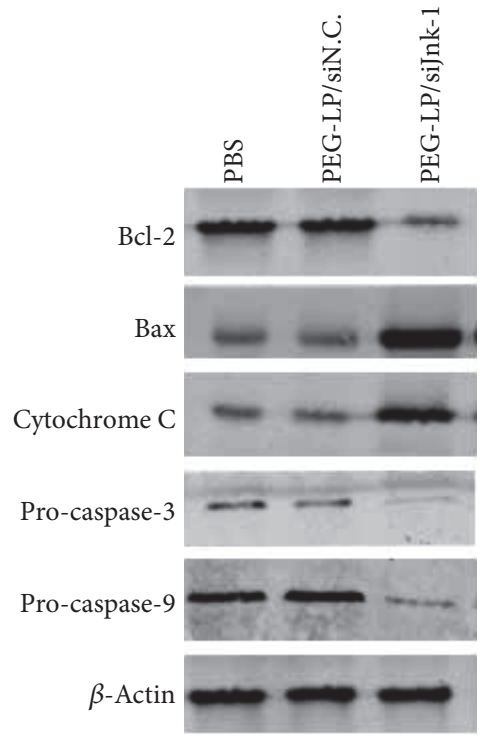

FIGURE 5: The apoptotic mechanisms of Jnk-1 knockdown in vitro. Western blot analysis of caspase activity and apoptotic related markers in DU145 cells treated with PBS, PEG-LP/siN.C. NPs, or PEG-LP/siJnk-1 NPs.

biodegradable PLGA polymer core containing PEI/siRNA, with the assistance of a cationic lipid DSPE-PEG. Such NPs exhibited high siRNA encapsulation efficiency. Meanwhile, the prepared PEG-LP/siRNA NPs could successfully enter cells, which in turn significantly downregulated the expression of its target genes in DU145 cells and induced apoptosis. Moreover, PEG-LP/siJnk-1 NPs could inhibit the tumor growth in a DU145 xenograft tumor model following systemic administration, suggesting its potential application for the delivery of siRNA.

\section{Conflicts of Interest}

There are no conflicts of interest to declare.

\section{Acknowledgments}

This work was financially supported by the Science and Technology Planning Project of Jilin Province (Grant no. 20160520166JH) and Health Technology Innovation Project of Jilin Province (Grant no. 2016J070). 


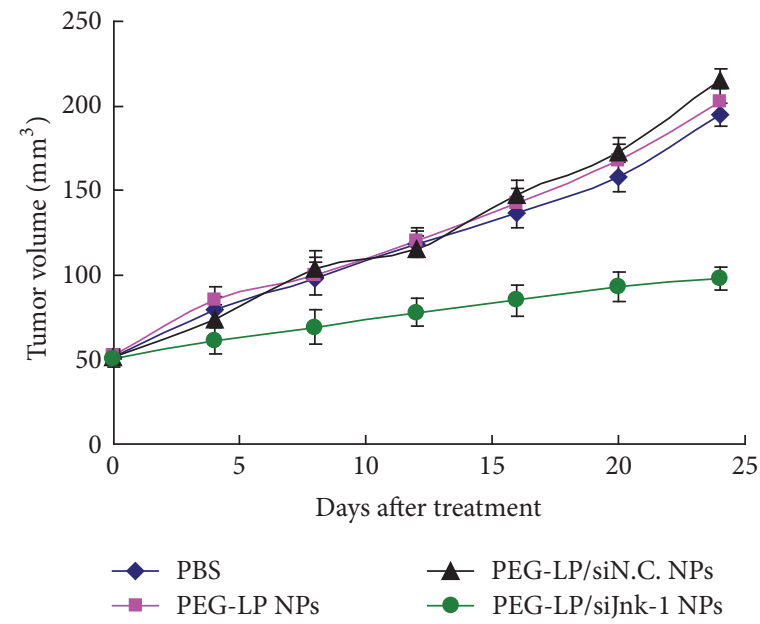

(a)

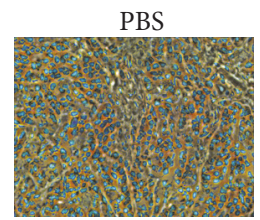

PEG-LP NPs

PEG-LP/siN.C. NPs PEG-LP/siJnk-1 NPs
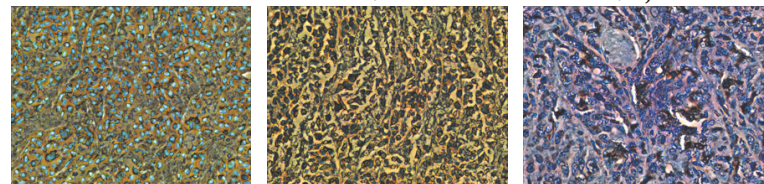

(b)

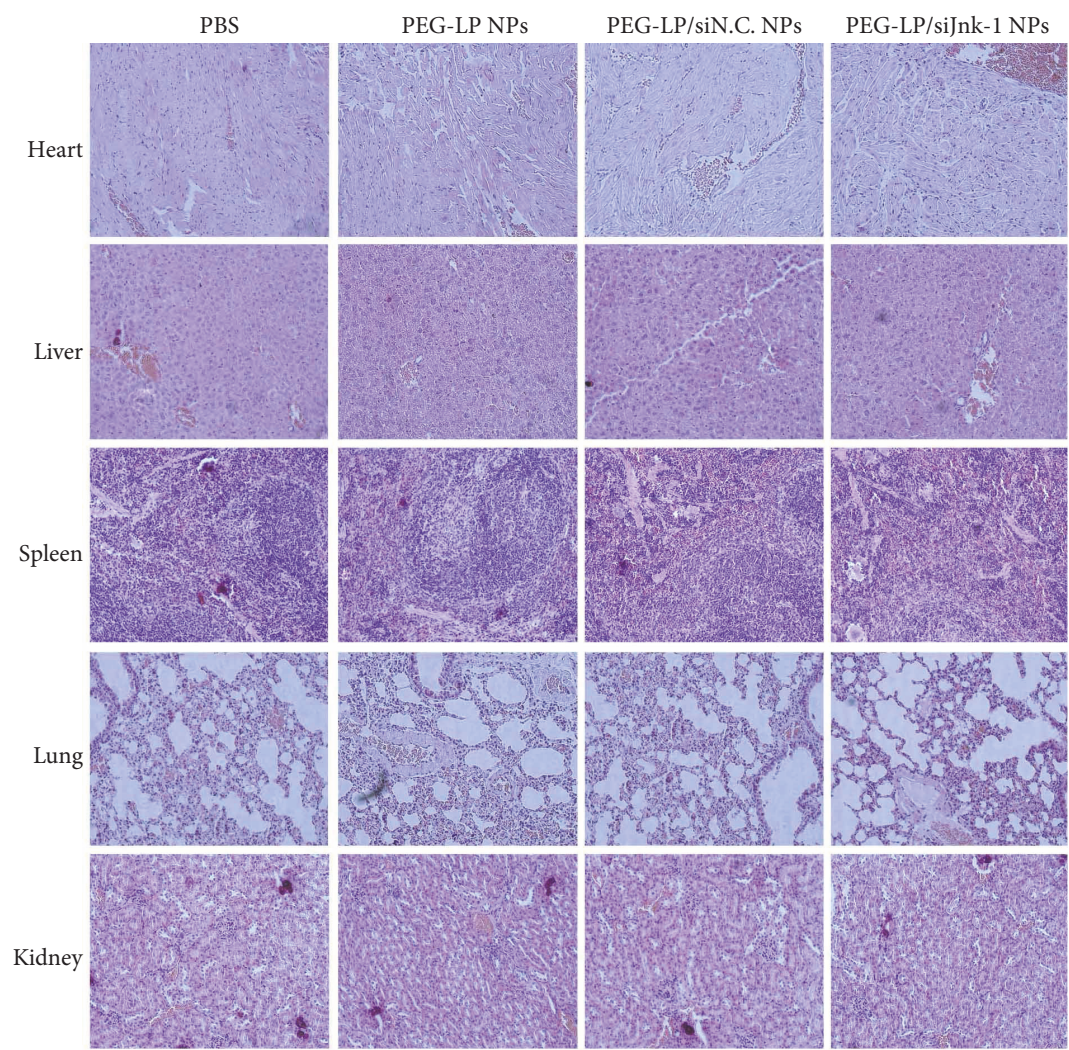

(c)

Figure 6: Systemic delivery of PEG-LP/siJnk-1 NPs suppressed the growth of DU145 xenografts in nude mice. (a) Relative tumor volume of nude mice bearing DU145 tumors treated with PBS, PEG-LP NPs, PEG-LP/siN.C. NPs, or PEG-LP/siJnk-1 NPs. Data are presented as the means \pm SD. (b) TUNEL assays of tumor tissues after treatment with the various formulations. (c) Hematoxylin and eosin (HE) staining of organs, including heart, liver, spleen, lung, and kidney from mice treated with the various formulations. 


\section{References}

[1] C. V. Pecot, G. A. Calin, R. L. Coleman, G. Lopez-Berestein, and A. K. Sood, "RNA interference in the clinic: challenges and future directions," Nature Reviews Cancer, vol. 11, no. 1, pp. 5967, 2011.

[2] K. A. Whitehead, R. Langer, and D. G. Anderson, "Knocking down barriers: advances in siRNA delivery," Nature Reviews Drug Discovery, vol. 8, no. 2, pp. 129-138, 2009.

[3] H. Takemoto, K. Miyata, S. Hattori et al., "Acidic pH-responsive siRNA conjugate for reversible carrier stability and accelerated endosomal escape with reduced IFN $\alpha$-associated immune response," Angewandte Chemie International Edition, vol. 52, no. 24, pp. 6218-6221, 2013.

[4] C. Yang, L. Nilsson, M. U. Cheema et al., "Chitosan/siRNA nanoparticles targeting cyclooxygenase type 2 attenuate unilateral ureteral obstruction-induced kidney injury in mice," Theranostics, vol. 5, no. 2, pp. 110-123, 2015.

[5] B. J. Hong, A. J. Chipre, and S. T. Nguyen, "Acid-degradable polymer-caged lipoplex (PCL) platform for siRNA delivery: Facile cellular triggered release of siRNA," Journal of the American Chemical Society, vol. 135, no. 47, pp. 17655-17658, 2013.

[6] A. Pichler, N. Zelcer, J. L. Prior, A. J. Kuil, and D. PiwnicaWorms, "In vivo RNA interference-mediated ablation of MDR1 P-glycoprotein," Clinical Cancer Research, vol. 11, no. 12, pp. 4487-4494, 2005.

[7] M. Everts, V. Saini, J. L. Leddon et al., "Covalently linked Au nanoparticles to a viral vector: Potential for combined photothermal and gene cancer therapy," Nano Letters, vol. 6, no. 4, pp. 587-591, 2006.

[8] T. Merdan, J. Kopeček, and T. Kissel, "Prospects for cationic polymers in gene and oligonucleotide therapy against cancer," Advanced Drug Delivery Reviews, vol. 54, no. 5, pp. 715-758, 2002.

[9] F. H. E. Schagen, M. Ossevoort, R. E. M. Toes, and R. C. Hoeben, "Immune responses against adenoviral vectors and their transgene products: A review of strategies for evasion," Critical Review in Oncology/Hematology, vol. 50, no. 1, pp. 5170, 2004.

[10] D. Omata, Y. Negishi, S. Hagiwara et al., "Enhanced gene delivery using Bubble liposomes and ultrasound for folate-PEG liposomes," Journal of Drug Targeting, vol. 20, no. 4, pp. 355-363, 2012.

[11] T. Ganbold, G. Gerile, H. Xiao, and H. Baigude, "Efficient in vivo siRNA delivery by stabilized d-peptide-based lipid nanoparticles," RSC Advances, vol. 7, no. 15, pp. 8823-8831, 2017.

[12] W. Hou, S. Wen, R. Guo, S. Wang, and X. Shi, "Partially acetylated dendrimer-entrapped gold nanoparticles with reduced cytotoxicity for gene delivery applications," Journal of Nanoscience and Nanotechnology, vol. 15, no. 6, pp. 4094-4105, 2015.

[13] J. Qiu, L. Kong, X. Cao, A. Li, H. Tan, and X. Shi, "Dendrimerentrapped gold nanoparticles modified with $\beta$-cyclodextrin for enhanced gene delivery applications," RSC Advances, vol. 6, no. 31, pp. 25633-25640, 2016.

[14] Y. Shan, T. Luo, C. Peng et al., "Gene delivery using dendrimerentrapped gold nanoparticles as nonviral vectors," Biomaterials, vol. 33, no. 10, pp. 3025-3035, 2012.
[15] T. Xiao, X. Cao, W. Hou, C. Peng, J. Qiu, and X. Shi, "Poly(amidoamine) dendrimers modified with 1,2-epoxyhexane or 1,2epoxydodecane for enhanced gene delivery applications," Journal of Nanoscience and Nanotechnology, vol. 15, no. 12, pp. 1013410140, 2015.

[16] X. Li, Y. Chen, M. Wang, Y. Ma, W. Xia, and H. Gu, "A mesoporous silica nanoparticle - PEI - Fusogenic peptide system for siRNA delivery in cancer therapy," Biomaterials, vol. 34, no. 4, pp. 1391-1401, 2013.

[17] M. Günther, J. Lipka, A. Malek, D. Gutsch, W. Kreyling, and A. Aigner, "Polyethylenimines for RNAi-mediated gene targeting in vivo and siRNA delivery to the lung," European Journal of Pharmaceutics and Biopharmaceutics, vol. 77, no. 3, pp. 438-449, 2011.

[18] D. Fischer, Y. Li, B. Ahlemeyer, J. Krieglstein, and T. Kissel, "In vitro cytotoxicity testing of polycations: influence of polymer structure on cell viability and hemolysis," Biomaterials, vol. 24, no. 7, pp. 1121-1131, 2003.

[19] Y. He, G. Cheng, L. Xie, Y. Nie, B. He, and Z. Gu, "Polyethyleneimine/DNA polyplexes with reduction-sensitive hyaluronic acid derivatives shielding for targeted gene delivery," Biomaterials, vol. 34, no. 4, pp. 1235-1245, 2013.

[20] X. Zhou, X. Li, M. Gou et al., "Antitumoral efficacy by systemic delivery of heparin conjugated polyethylenimineplasmid interleukin-15 complexes in murine models of lung metastasis," Cancer Science, vol. 102, no. 7, pp. 1403-1409, 2011.

[21] C. Cai, Y. Xie, L. Wu et al., "PLGA-based dual targeted nanoparticles enhance miRNA transfection efficiency in hepatic carcinoma," Scientific Reports, vol. 7, Article ID 46250, 2017.

[22] A. M. Aldayel, Y. W. Naguib, H. L. O’Mary et al., "Acid-Sensitive Sheddable PEGylated PLGA Nanoparticles Increase the Delivery of TNF- $\alpha$ siRNA in Chronic Inflammation Sites," Molecular Therapy - Nucleic Acids, vol. 5, p. e340, 2016.

[23] C. R. Weston and R. J. Davis, "The JNK signal transduction pathway," Current Opinion in Cell Biology, vol. 19, no. 2, pp. 142149, 2007.

[24] V. Sehgal and P. T. Ram, "Network Motifs in JNK Signaling," Genes \& Cancer, vol. 4, no. 9-10, pp. 409-413, 2013.

[25] K.-I. Matsuda, A. Sato, M. Okada et al., "Targeting JNK for therapeutic depletion of stem-like glioblastoma cells," Scientific Reports, vol. 2, article no. 516, 2012.

[26] E. Parra and J. Ferreira, "Knockdown of the c-Jun-N-terminal kinase expression by siRNA inhibits MCF-7 breast carcinoma cell line growth," Oncology Reports, vol. 24, no. 5, pp. 1339-1345, 2010.

[27] F. Dai and W. Liu, "Enhanced gene transfection and serum stability of polyplexes by PDMAEMA-polysulfobetaine diblock copolymers," Biomaterials, vol. 32, no. 2, pp. 628-638, 2011.

[28] M. L. Würstle and M. Rehm, "A systems biology analysis of apoptosome formation and apoptosis execution supports allosteric procaspase- 9 activation," The Journal of Biological Chemistry, vol. 289, no. 38, pp. 26277-26289, 2014. 


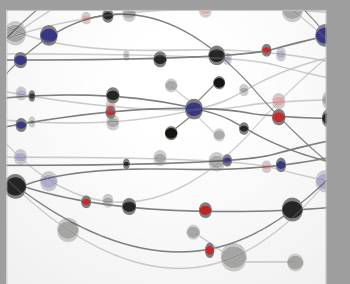

The Scientific World Journal
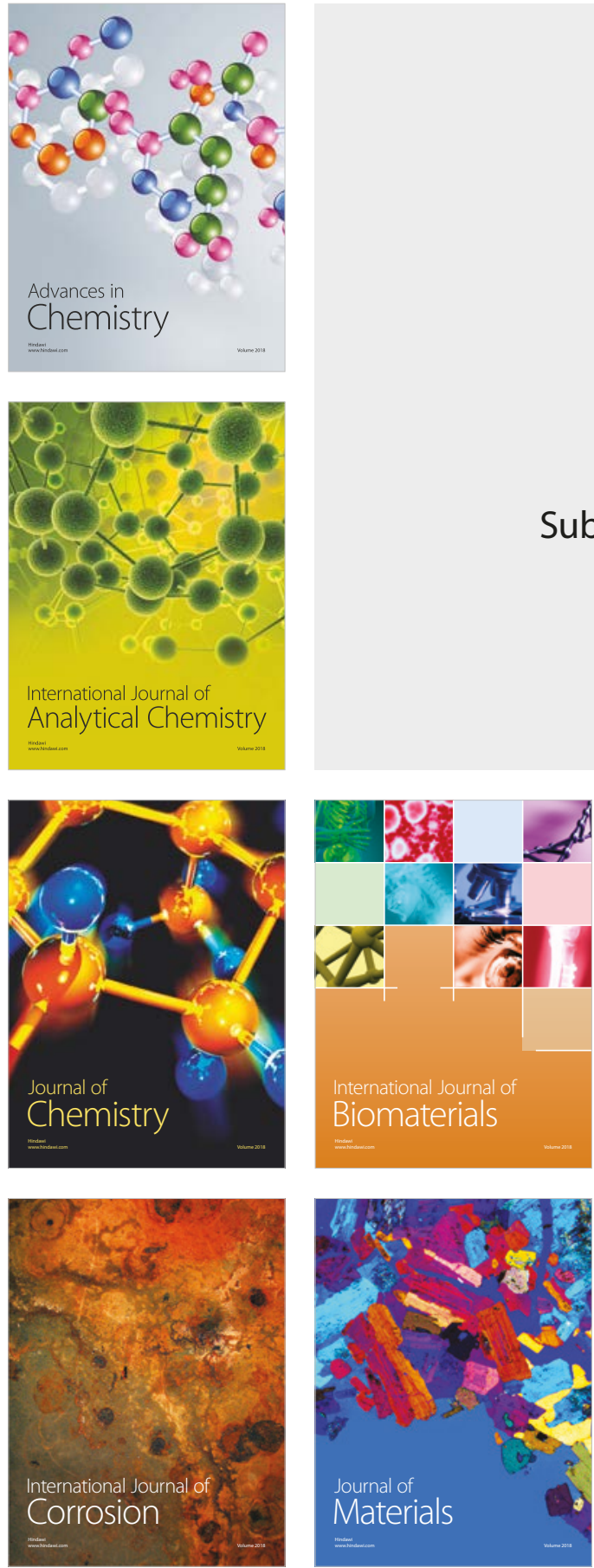

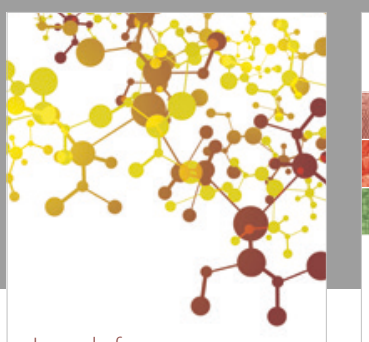

Journal of

Applied Chemistry
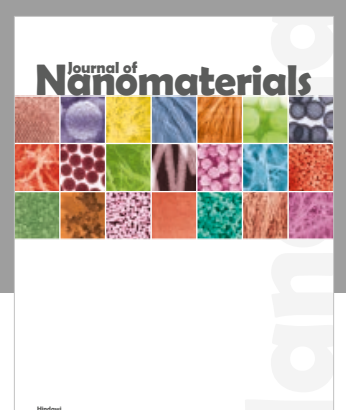

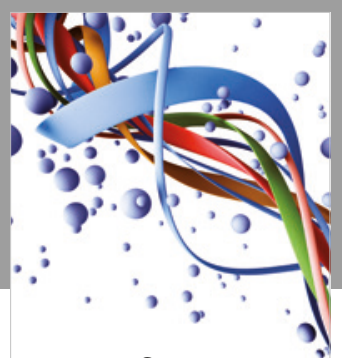

Scientifica

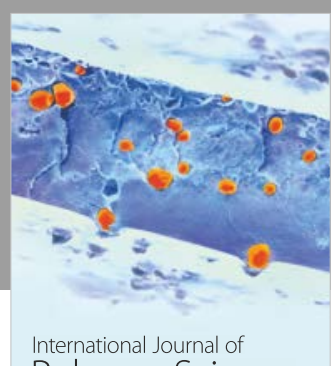

Polymer Science

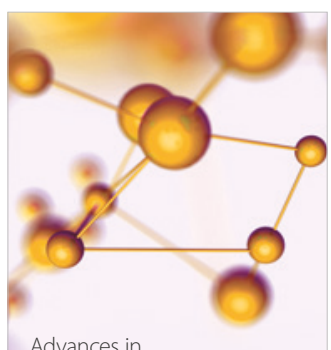

Physical Chemistry
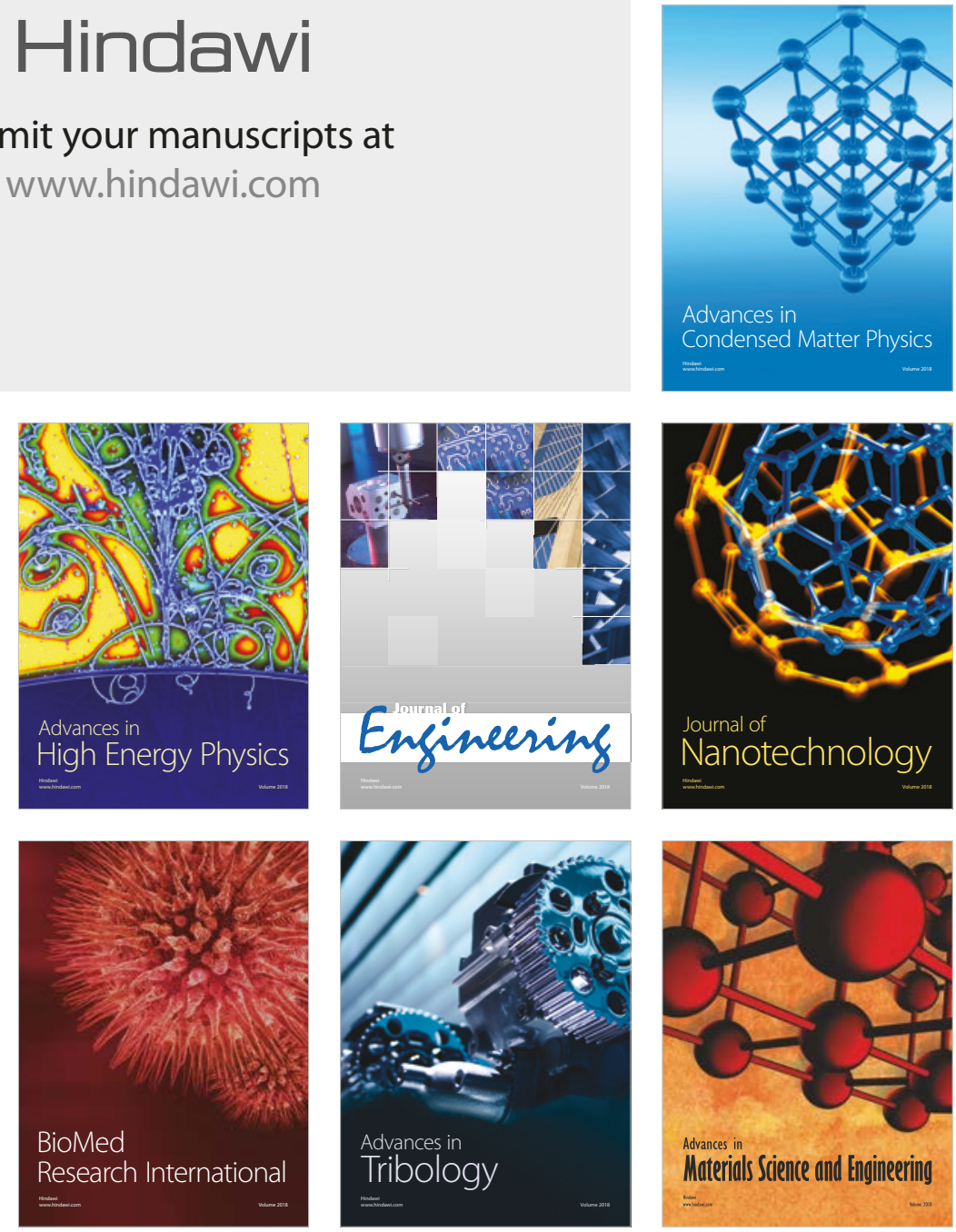